(2) OPEN ACCESS

\title{
Improving the diagnosis of heart failure in patients with atrial fibrillation
}

\author{
Karina V Bunting (D) , ${ }^{1,2}$ Simrat K Gill, ${ }^{1,2}$ Alice Sitch, ${ }^{3,4}$ Samir Mehta, ${ }^{5}$ Kieran O'Connor, ${ }^{2}$ \\ Gregory YH Lip (D) , ${ }^{6,7}$ Paulus Kirchhof (D) , 1,8 Victoria Y Strauss, ${ }^{9}$ Kazem Rahimi, ${ }^{10,11}$ \\ A John Camm (D) ,' ${ }^{12}$ Mary Stanbury, ${ }^{13}$ Michael Griffith, ${ }^{2}$ Jonathan N Townend, ${ }^{1,2}$ \\ Georgios V Gkoutos, ${ }^{14,15}$ Andreas Karwath, ${ }^{14}$ Richard P Steeds (D) , 1,2 \\ Dipak Kotecha (D) , 1,2,15 on behalf of the RAte control Therapy Evaluation in permanent \\ Atrial Fibrillation (RATE-AF) trial group
}

\begin{abstract}
- Additional material is published online only. To view please visit the journal online (http://dx.doi.org/10.1136/ heartjnl-2020-318557).

For numbered affiliations see end of article.
\end{abstract}

\section{Correspondence to} Professor Dipak Kotecha, University of Birmingham Institute of Cardiovascular Sciences, Birmingham, Birmingham, UK; d.kotecha@bham.ac.uk

RPS and DK are joint senior authors.

Received 30 October 2020 Revised 21 January 2021 Accepted 25 January 202 Published Online First 10 March 2021

\section{Linked}

- http://dx.doi.org/10.1136/ heartjnl-2020-318923

Check for updates

(C) Author(s) (or their employer(s)) 2021. Re-use permitted under CC BY. Published by BMJ.

To cite: Bunting $\mathrm{KV}$ Gill SK, Sitch A, et al. Heart 2021:107:902-908.

\section{ABSTRACT}

Objective To improve the echocardiographic assessment of heart failure in patients with atrial fibrillation (AF) by comparing conventional averaging of consecutive beats with an index-beat approach, whereby measurements are taken after two cycles with similar R-R interval.

Methods Transthoracic echocardiography was performed using a standardised and blinded protocol in patients enrolled in the RATE-AF (RAte control Therapy Evaluation in permanent Atrial Fibrillation) randomised trial. We compared reproducibility of the index-beat and conventional consecutive-beat methods to calculate left ventricular ejection fraction (LVEF), global longitudinal strain (GLS) and E/e' (mitral E wave max/ average diastolic tissue Doppler velocity), and assessed intraoperator/interoperator variability, time efficiency and validity against natriuretic peptides.

Results 160 patients were included, $46 \%$ of whom were women, with a median age of 75 years (IQR 69-82) and a median heart rate of 100 beats per minute (IQR 86-112). The index-beat had the lowest withinbeat coefficient of variation for LVEF (32\%, vs $51 \%$ for 5 consecutive beats and $53 \%$ for 10 consecutive beats), GLS ( $26 \%$, vs $43 \%$ and $42 \%)$ and E/e' (25\%, vs $41 \%$ and $41 \%)$. Intraoperator $(n=50)$ and interoperator $(n=18)$ reproducibility were both superior for index-beats and this method was quicker to perform ( $p<0.001)$ : 35.4 s to measure E/e' (95\% Cl 33.1 to 37.8$)$ compared with $44.7 \mathrm{~s}$ for 5-beat $(95 \% \mathrm{Cl} 41.8$ to 47.5$)$ and $98.1 \mathrm{~s}$ for 10-beat ( $95 \%$ Cl 91.7 to 104.4) analyses. Using a single index-beat did not compromise the association of LVEF, GLS or E/e' with natriuretic peptide levels.

Conclusions Compared with averaging of multiple beats in patients with $A F$, the index-beat approach improves reproducibility and saves time without a negative impact on validity, potentially improving the diagnosis and classification of heart failure in patients with AF.

\section{INTRODUCTION}

The prevalence of atrial fibrillation (AF) is climbing, ${ }^{1}$ and around $50 \%$ of patients with $\mathrm{AF}$ have or will develop heart failure. ${ }^{2} 3$ Assessment of systolic and diastolic ventricular function using echocardiography is essential for AF management, including stroke risk stratification, choice of rate and rhythm control therapy, and the identification of heart failure. ${ }^{4}$ However, assessment of systolic and diastolic function is challenging in AF due to variable R-R intervals leading to beat-to-beat changes in left ventricular function. This may explain why the association of echocardiography parameters with adverse events is weaker in AF compared with sinus rhythm. ${ }^{5}$ In clinical practice, multiple beats are averaged despite a lack of any evidence base for this approach. ${ }^{6}$ Multiple beat acquisition and analysis is time-consuming and may not adequately compensate for the variable stroke volume and filling time seen in $\mathrm{AF}^{7}$ To correctly classify heart failure in the context of AF and manage it appropriately, it is crucial to have accurate and reproducible measurements of systolic and diastolic function. ${ }^{8}$

The aim of this study was to systematically compare the reproducibility and efficiency of a more physiological approach to improve the assessment of heart failure in patients with AF. The indexbeat method takes into account time-dependent processes involved in contractility and relaxation. ${ }^{9}$ We compared this approach with conventional averaging of 3 beats (clinical routine) and 5 beats and 10 beats (as recommended in guidelines), with complete blinding of observers. Unlike previous studies, there was no preselected exclusion of patients according to image quality. ${ }^{10}$ We hypothesised that the index-beat method would be more reproducible and time-efficient, facilitating better management of patients with $\mathrm{AF}$ and heart failure.

\section{METHODS}

\section{Patient population}

Baseline echocardiograms were evaluated for al participants enrolled in the RATE-AF (RAte control Therapy Evaluation in permanent Atrial Fibrillation) randomised trial after obtaining written informed consent (NCT02391337). The trial received oversight from a trial steering committee and data monitoring committee with independent chairs. The rationale and methods for the RATE-AF trial have previously been published. ${ }^{11}$ In brief, outpatients aged 60 years or older with permanent 
AF and symptoms of heart failure (New York Heart Association class II or above) were randomised to digoxin or beta-blockers for rate control of AF. Permanent AF was defined as a physician's decision for rate control and no plans for antiarrhythmic drugs or interventional rhythm control. Patients were excluded if their heart rate was $<60$ beats per minute or had prior evidence of second-degree or third-degree heart block. Other exclusion criteria were minimised to enable generalisable 'real-world' results. There were no exclusion criteria related to known heart failure or according to left ventricular ejection fraction (LVEF), apart from those with decompensated heart failure in the last 14 days, evidenced by the need for intravenous inotropes, vasodilators or diuretics. ${ }^{11}$ The trial was publicly funded by the UK National Institute for Health Research and the main results have been published. ${ }^{10}$

\section{Echocardiography protocol}

AF was confirmed on 12-lead ECG. All patients then underwent transthoracic echocardiography using Philips EPIQ 7 and X5-1 transducer, by an experienced echocardiographer accredited with the British Society of Echocardiography. Patients were positioned in left lateral decubitus position and images were acquired during quiet respiration according to an echocardiography protocol ratified prior to the trial commencing.

A minimum of 30-beat loops were obtained of the apical twochamber, three-chamber and four-chamber views. All images were optimised to maximise frame rate while ensuring all left ventricular segments were visible. A minimum of 30 traces of tissue Doppler imaging (TDI)-derived lateral and septal e' were obtained and mitral inflow using pulse wave Doppler acquired at a sweep speed of $50 \mathrm{~mm} / \mathrm{s}$. The recorded images were given a unique identification number and all patient-identifiable features (including trial number) were removed, blinding the operator to patient, trial and clinical details.

All measurements were analysed offline by the same operator a minimum of 3 months after the scan date. To minimise selection bias, there were no predefined exclusions made on the quality of imaging; however, if image quality was insufficient to accurately obtain measurements, these images were excluded from analysis. All analyses were performed on Philips Q-Station (V.3.5; Philips Healthcare, Andover, Massachusetts). For LVEF, Simpson's biplane LVEF was measured from the apical fourchamber and two-chamber views. Longitudinal strain was taken from the apical two-chamber, three-chamber and four-chamber views and then averaged to generate overall global longitudinal strain (GLS). The mean frame rate for GLS acquisition was 57 Hertz (SD 7.0). For E/e', mitral valve peak E velocity was measured and then divided by the TDI-derived e' (averaged from the lateral and septal walls).

\section{Index-beat and conventional averaging method}

The index-beat was identified as the cardiac cycle which followed a preceding and pre-preceding R-R interval of similar duration (within $60 \mathrm{~ms}$ of each other; figure 1). R-R intervals were measured using a calliper in the reporting software. The index-beats were selected consecutively from the beginning of each set of echocardiogram data to avoid selection bias. Conventional analysis involved averaging of 3,5 and 10 consecutive cardiac cycles from a 10-beat data set.

\section{Intraoperator reproducibility}

The same operator took a second set of 10 images to measure GLS and E/e' (using the methods described above) with a single index-beat and an average of 3, 5 and 10 beats.

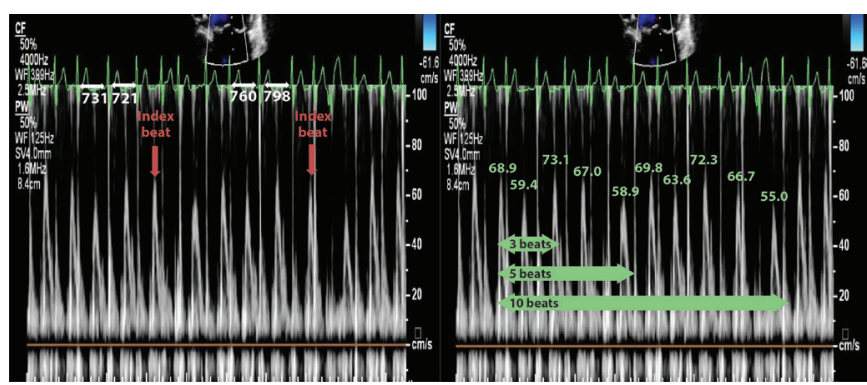

Figure 1 Index-beat approach versus conventional averaging of consecutive beats. Left panel: pulse wave mitral inflow Doppler using the index-beat method, with similar preceding and pre-preceding R-R intervals indicated by the red arrows. Values are the R-R interval length of the preceding cardiac cycle. Right panel: 3, 5 and 10 consecutive beats. Values are the peak E velocity measurement in $\mathrm{cm} / \mathrm{s}$.

\section{Interoperator reproducibility and time comparison}

A second accredited operator obtained images in 18 randomly selected patients to measure GLS and E/e' using a single indexbeat and average of 3,5 and 10 beats. The operator was blinded to the previously recorded measurements as well as all clinical details of each echocardiogram. The time taken to select and measure E/e' using a single index-beat and guidelinerecommended 5 and 10 consecutive beats was measured, with time commencing from the first visualisation of Doppler images.

\section{Validity}

All patients had N-terminal pro-B-type natriuretic peptide (NT-proBNP) measured on the same day as their echocardiogram using an Abbott Alinity platform. This was correlated with the parameters LVEF Simpson's biplane, GLS and E/e'.

\section{Patient and public involvement}

A patient and public involvement team were involved in the design and conduct of this study and assisted with the plain English summary.

\section{Statistical analysis}

Summary results are presented as percentage, mean with SD, or median with IQR (displayed as 25 th-75th quartiles). All echocardiographic measurements were transformed to their natural $\log$ value. A multilevel mixed effects linear regression model (adjusting for the random effects of the patient) was used to calculate the intraclass correlation coefficient (ICC) and derive the SD $(\sigma)$, with $95 \%$ CI for the within-beat variability. Post-hoc analysis indicated $>90 \%$ assurance that the sample size was sufficient for ICC comparison. ${ }^{12}$ The coefficient of variation (CV) within three index-beats and within each set of consecutive beats (3, 5 and 10) was calculated using the following formula: $\sqrt{\exp }\left(\mathrm{SD}^{2}\right)-1 \times 100 ; 95 \%$ CIs were calculated for each $\mathrm{CV}$. For intraoperator and interoperator reproducibility, Bland and Altman analysis was used to obtain the mean bias and limits of agreement, and the ICC with 95\% CI was calculated at the patient level for each measurement method using a multilevel mixed effects linear regression model (adjusting for the random effects of the patient and the time the measurement was taken). The statistical comparison of time taken was performed using a two-tailed t-test accounting for unequal variance. The association with NT-proBNP was compared using Spearman's $r$ and univariate linear regression analysis. The difference in correlation coefficient between measuring on an index-beat and 


\begin{tabular}{|c|c|}
\hline Characteristics & $\mathrm{N}=160$ \\
\hline Age, median years (IQR) & $75(69-82)$ \\
\hline Women, $\mathrm{n}(\%)$ & $74(46)$ \\
\hline Years in $A F$, mean years (SD) & $3.8(6)$ \\
\hline Ethnicity: white British or Irish, $\mathrm{n}(\%)$ & $149(93.1)$ \\
\hline Ethnicity: black African, Caribbean or black British, $\mathrm{n}(\%)$ & $3(1.9)$ \\
\hline Ethnicity: Asian or Asian British, $\mathrm{n}(\%)$ & $8(5.01)$ \\
\hline Previous rhythm control, n (\%) & $23(14)$ \\
\hline Modified EHRA class 3 or $4, n(\%)$ & $77(48)$ \\
\hline Previous heart failure clinical diagnosis, $\mathrm{n}(\%)$ & $59(37)$ \\
\hline Signs of heart failure at randomisation, $\mathrm{n}(\%)$ & $84(53)$ \\
\hline NYHA class III or IV, n (\%) & $61(38)$ \\
\hline Previous myocardial infarction, $\mathrm{n}(\%)$ & $13(8)$ \\
\hline Previous stroke, $\mathrm{n}(\%)$ & $19(12)$ \\
\hline Previous TIA, n (\%) & $15(9)$ \\
\hline COPD, n (\%) & $29(18)$ \\
\hline Diabetes mellitus, $\mathrm{n}(\%)$ & $38(24)$ \\
\hline Heart rate, median bpm (IQR) & $100(86-112)$ \\
\hline Systolic BP, median mm Hg (IQR) & $134(123-148)$ \\
\hline Diastolic BP, median mm Hg (IQR) & $84(76-93)$ \\
\hline Body mass index, median $\mathrm{kg} / \mathrm{m}^{2}$ (IQR) & $30(26-34)$ \\
\hline NT-proBNP, median pg/mL (IQR) & $1057(744-1522)$ \\
\hline Estimated GFR, median mL/min (IQR) & $67(55-77)$ \\
\hline Already receiving anticoagulant medication, $\mathrm{n}(\%)$ & $135(84)$ \\
\hline Antihypertensive medication, $\mathrm{n}(\%)$ & $116(73)$ \\
\hline Inhalers for airway disease, $\mathrm{n}(\%)$ & $40(25)$ \\
\hline \multicolumn{2}{|c|}{$\begin{array}{l}\text { AF, atrial fibrillation; BP, blood pressure; bpm, beats per minute; COPD, chronic } \\
\text { obstructive pulmonary disorder; EHRA, European Heart Rhythm Association; GFR, } \\
\text { glomerular filtration rate; NT-proBNP, N-terminal pro-B-type natriuretic peptide; } \\
\text { NYHA, New York Heart Failure Association functional classification; TIA, transient } \\
\text { ischaemic attack. }\end{array}$} \\
\hline
\end{tabular}

averaging 3, 5 and 10 consecutive beats was calculated using the method of Meng et al. ${ }^{13}$ Statistical analysis was performed using Stata V.14.2. A two-tailed p value of 0.05 was considered statistically significant.

\section{RESULTS}

One hundred and sixty patients were included, with a median age of 75 years (IQR 69-82), heart rate of 100 beats per minute at time of acquisition (86-112) and blood pressure of 134/84 mm Hg (123/76-148/93) (see table 1). The median LVEF was $59 \%$ (52-64), GLS -14\% (-12 to -15$)$ and E/e' 9.4 (7.8-11.7); variables measured by consecutive beats are listed in online supplemental table 2. Image quality was insufficient for LVEF measurement in 18 patients (11.3\%) and for GLS in 21 patients (13.1\%), with strain assessment more challenging at higher heart rates. All patients had sufficient image quality to measure E/e'. Other echocardiogram parameters of interest are summarised in table 2. Online supplemental figure 1 depicts the flow diagram of the study population. A plain English summary of results is presented in online supplemental table 1 .

\section{Within-beat reproducibility}

The index-beat approach had less variability compared with conventional consecutive beat averaging for all comparisons of CV for LVEF, GLS and E/e' (figure 2 and table 3). In all cases, the difference between the index-beat method and the variability of 3,5 and 10 consecutive beats was statistically significant with no overlap of $95 \%$ CIs. Similarly, the index-beat
Table 2 Echocardiography parameters

\begin{tabular}{lc}
\hline Echocardiographic measurement & Baseline \\
\hline Left ventricular end diastolic volume, median mL (IQR) & $76(57-99)$ \\
\hline Left ventricular end systolic volume, median mL (IQR) & $30(22-42)$ \\
Stroke volume, median mL (IQR) & $55(45-64)$ \\
\hline Left ventricular ejection fraction, median \% (IQR) & $59(52-64)$ \\
Global longitudinal strain, median \% (IQR) & $-14(-12$ to -15) \\
\hline Lateral s', median cm/s (IQR) & $6.7(5.6-7.9)$ \\
\hline Septal s', median cm/s (IQR) & $6.1(5.1-7.2)$ \\
\hline Average e', median cm/s (IQR) & $9.3(8.1-10.9)$ \\
\hline Mitral E velocity, median cm/s (IQR) & $89.7(77.1-102.8)$ \\
\hline Mitral deceleration time, median ms (IQR) & $212(188-234)$ \\
\hline Average Ele', median (IQR) & $9.4(7.8-11.7)$ \\
\hline Isovolumic relaxation time, median ms (IQR) & $97(89-108)$ \\
\hline Pulmonary vein ratio, mean (SD) & $0.7(0.1)$ \\
\hline Pulmonary vein deceleration time, median (IQR) & $242(223-258)$ \\
\hline Left atrial volume indexed to BSA, median mL/m² (IQR) & $38(32-49)$ \\
\hline Left atrial ejection fraction, median \% (IQR) & $23(15-33)$ \\
\hline TAPSE mm, median (IQR) & $18.7(17.1-21.8)$ \\
\hline BSA, body surface area: TAPSE, tricuspid annular plane systolic excursion
\end{tabular}

BSA, body surface area; TAPSE, tricuspid annular plane systolic excursion.

approach demonstrated higher ICC values for LVEF, GLS and E/e' (figure 3 and table 3).

For LVEF, the index-beat approach had the smallest CV of $32 \%(95 \%$ CI 31 to 34$)$ and strongest ICC of 0.94 (0.93 to 0.96 ), compared with $54 \%$ (52 to 57 ) and 0.71 (0.64 to 0.77$)$ for 3 consecutive beats, $51 \%$ (50 to 53 ) and 0.76 (0.71 to 0.81 ) for 5 consecutive beats, and 53\% (52 to 54) and 0.74 (0.69 to $0.79)$ for 10 consecutive beats. The mean RR1 of the indexbeat (pre-preceding R-R interval) was $552 \mathrm{~ms}$ and the mean RR2 (preceding R-R interval) was $555 \mathrm{~ms}$, with a mean RR1 to RR2 ratio of 1.00 .

For GLS, the index-beat approach had the smallest CV of $26 \%$ (95\% CI 25 to 27) and strongest ICC of 0.88 (0.85 to 0.91 ), compared with $44 \%$ (42 to 46 ) and 0.81 (0.76 to 0.85 ) for 3 consecutive beats, $43 \%$ ( 42 to 44 ) and 0.82 (0.77 to 0.85 ) for 5 consecutive beats, and $42 \%$ (41 to 43$)$ and 0.80 (0.75 to 0.84$)$ for 10 consecutive beats. The mean RR1 of the index-beat was

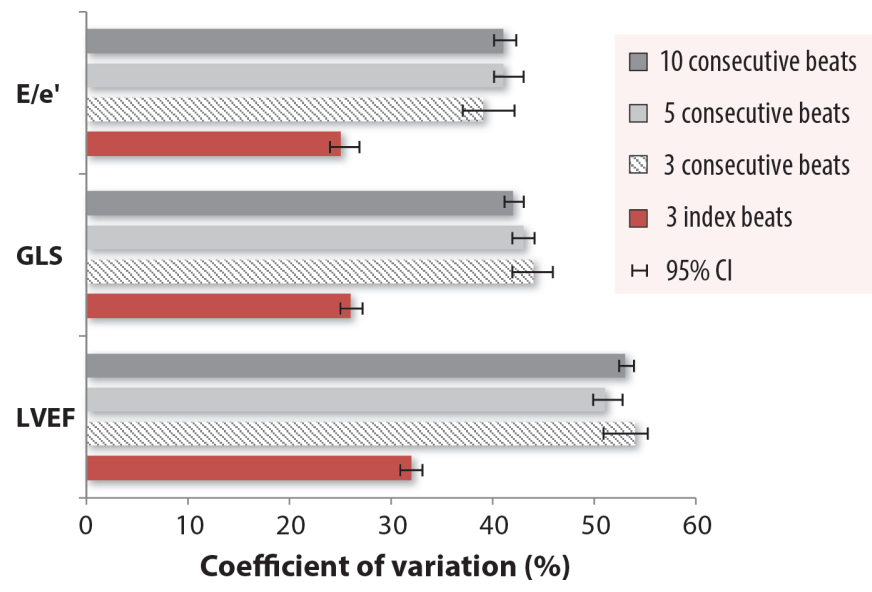

Figure 2 Comparison of the coefficient of variation of within-beat variability. Within-beat coefficient of variation for Simpson's biplane LVEF, GLS and E/e' between 3 index-beats, compared with 3, 5 and 10 consecutive beats with $95 \% \mathrm{Cl}$. E/e', mitral E wave max/average diastolic tissue Doppler velocity from the septal and lateral annulus; GLS, global longitudinal strain; LVEF, left ventricular ejection fraction. 
Table 3 Within-beat variability of 3 index-beats versus 3, 5 and 10 consecutive beats

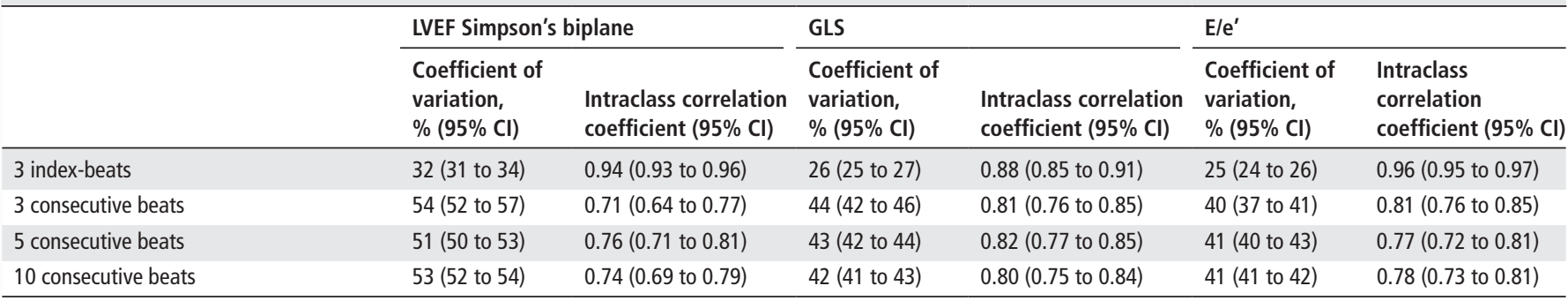

E/e', mitral E wave max/average diastolic tissue Doppler velocity from the septal and lateral annulus; GLS, global longitudinal strain; LVEF, left ventricular ejection fraction.

$568 \mathrm{~ms}$ and the mean RR2 was $570 \mathrm{~ms}$, with a mean RR1 to RR2 ratio of 1.09 .

For E/e', the index-beat approach had the smallest CV of $25 \%$ (95\% CI 24 to 26) and strongest ICC of 0.96 (0.95 to 0.97$)$, compared with $40 \%$ (37 to 41 ) and 0.81 (0.76 to 0.85$)$ for the average of 3 beats, $41 \%$ (40 to 43 ) and 0.77 (0.72 to 0.81 ) for the average of 5 beats, and $41 \%$ (41 to 42$)$ and 0.78 ( 0.73 to $0.81)$ for the average of 10 beats. The mean RR1 of the indexbeat was $653 \mathrm{~ms}$ and the mean RR2 was $655 \mathrm{~ms}$, with a mean RR1 to RR2 ratio of 1.00 .

\section{Intraoperator and interoperator reproducibility}

Intraoperator reproducibility of the index-beat method compared with consecutive beat averaging was assessed in 50 patients for E/e' and GLS (with similar patient characteristics to the main cohort; online supplemental table 3). For E/e', the index-beat had the smallest bias at -0.2 with similar limits of agreement to the average of 10 beats $(-4.2$ to 3.9$)$ and the highest ICC of 0.91 (95\% CI 0.84 to 0.95 ) (table 4 and online supplemental figure 2). Similar findings were seen for GLS, with the indexbeat method having the smallest bias at -0.5 with narrow limits of agreement ( -3.6 to 2.6) and the highest ICC of 0.82 (95\% CI 0.72 to 0.90 ) (online supplemental table 4 and online supplemental figure 2).

Interoperator reproducibility was tested in 18 randomly selected patients for GLS and E/e', with a new set of images taken by a second operator (with similar patient characteristics as seen in online supplemental table 3). The index-beat demonstrated only a small degree of bias for both GLS and E/e'. ICC values for the index-beat were 0.72 for GLS (95\% CI 0.45 to 0.88 ) and 0.94 for E/e' (95\% CI 0.87 to 0.98 ) (table 4, online supplemental table 4 and supplemental figure 3). Comparison of the second operator's assessment of E/e' with measurements taken by the first operator (from a different set of images) demonstrated that the index-beat method had similar or higher levels of reproducibility than averaging 5 or 10 consecutive beats: Bland and Altman bias (limits of agreement) of -0.6 ( -3.5 to 4.6$)$, vs 0.8 for 5 beats $(-3.3$ to 4.9$)$ and 0.4 for 10 beats $(-2.7$ to 3.6$)$.

\section{Efficiency and validity of the index-beat method}

The index-beat method took significantly less time to measure E/e' (mean 35.4 s; 95\% CI 33.1 to 37.8 ), compared with averaging 5 consecutive beats $(44.7 \mathrm{~s} ; 41.8$ to $47.5 ; \mathrm{p}<0.001)$ or 10 consecutive beats $(98.1 \mathrm{~s} ; 91.7$ to $104.4 ; \mathrm{p}<0.001)$ (figure 3 and table 4). Using the index-beat method saved 9.3 and $62.7 \mathrm{~s}$, respectively (absolute difference in means).

Despite only using a single index-beat, there was no evidence that this impacted on the validity; there were no significant differences in the correlation of NT-proBNP with echocardiographic variables when comparing index-beats with conventional averaging (figure 3). For example, the correlation of LVEF with NT-proBNP was 0.11 using a single index-beat
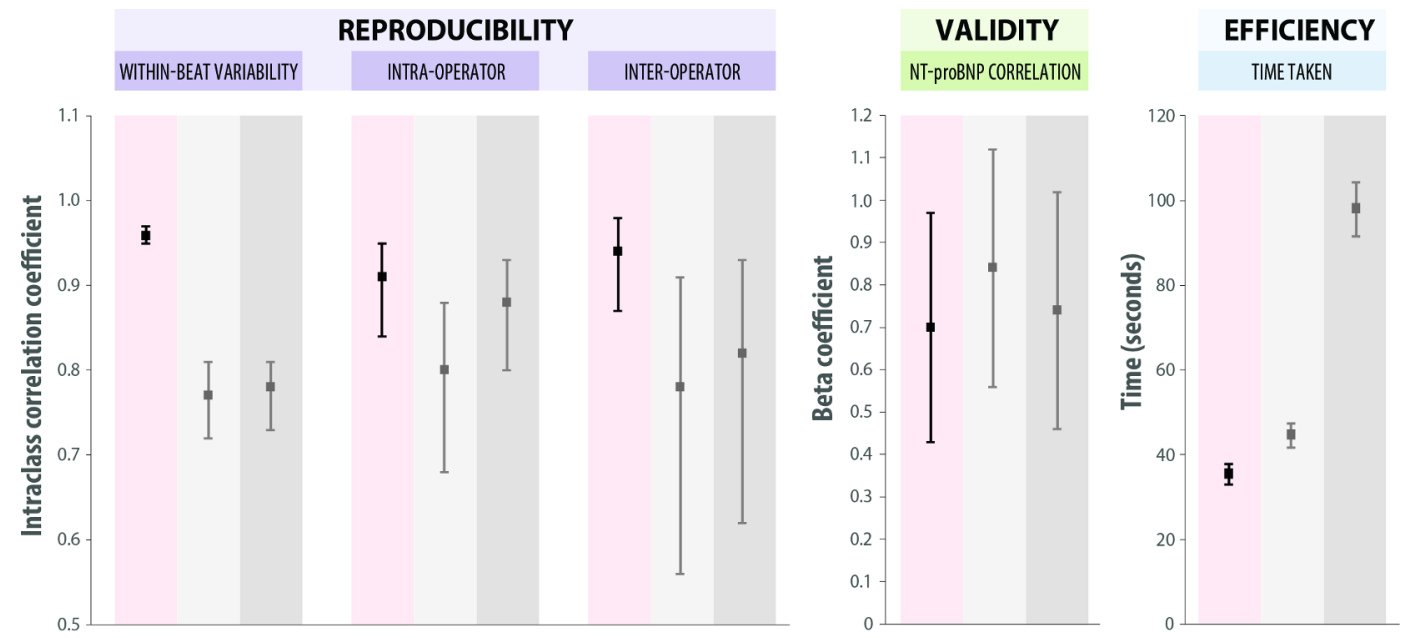

Key:

- Index beat

- 5 consecutive beats

10 consecutive beats

Figure 3 Reproducibility, validity and time efficiency of the index-beat approach for E/e'. Comparison of the index-beat method verses averaging of 5 and 10 consecutive beats for: (1) Reproducibility (ICC and 95\% Cl for within-beat, intra- and inter-operator variability); (2) Validity (beta coefficient for NT-proBNP with 95\% Cl); and (3) Time taken to measure E/e' (mean seconds, with 95\% Cl). E/e', mitral E wave max / average diastolic tissue Doppler velocity from the septal and lateral annulus; ICC, intraclass correlation coefficient; NT-proBNP, N-terminal pro-B-type natriuretic peptide. 
Table 4 Intraoperator and interoperator reproducibility and time efficiency for E/e'

\begin{tabular}{|c|c|c|c|c|c|}
\hline & \multicolumn{2}{|c|}{ Intraoperator reproducibility, $\mathrm{n}=50$} & \multicolumn{2}{|c|}{ Interoperator reproducibility, $\mathrm{n=18}$} & \multirow{2}{*}{$\begin{array}{l}\text { Time taken }(\mathrm{s}) \text { to } \\
\text { select and measure } \\
\text { E/e' }(95 \% \mathrm{Cl}), \mathrm{n}=18\end{array}$} \\
\hline & $\begin{array}{l}\text { Bias (limits of } \\
\text { agreement) }\end{array}$ & ICC $(95 \% \mathrm{Cl})$ & $\begin{array}{l}\text { Bias (limits of } \\
\text { agreement) }\end{array}$ & ICC $(95 \% \mathrm{Cl})$ & \\
\hline Single index-beat & $-0.2(-4.2$ to 3.9$)$ & 0.91 (0.84 to 0.95$)$ & $-0.3(-2.9$ to 2.2$)$ & 0.94 (0.87 to 0.98 ) & 35.4 (33.1 to 37.8$)$ \\
\hline 3 consecutive beats & $-0.7(-6.2$ to 4.8$)$ & 0.74 (0.60 to 0.85 ) & $-1.1(-5.4$ to 3.2$)$ & 0.83 (0.64 to 0.93 ) & Not performed \\
\hline 5 consecutive beats & $-0.6(-5.3$ to 4.2$)$ & 0.80 (0.68 to 0.88 ) & $-1.1(-6.5$ to 4.2$)$ & 0.78 (0.56 to 0.91$)$ & 44.7 (41.8 to 47.5$)$ \\
\hline 10 consecutive beats & $-0.4(-4.2$ to 3.4$)$ & 0.88 (0.80 to 0.93 ) & $-0.9(-6.1$ to 4.2$)$ & 0.82 (0.62 to 0.93 ) & 98.1 (91.7 to 104.4$)$ \\
\hline
\end{tabular}

E/e', mitral E wave max/average diastolic tissue Doppler velocity from the septal and lateral annulus; ICC, intraclass correlation coefficient.

$(p<0.001)$ vs 0.10 for 10 consecutive beats $(p<0.001)$, with no significant difference between the two methods $(\mathrm{p}=0.84)$. Additional correlations for LVEF, GLS and E/e' are presented in online supplemental table 5. Using the index-beat method led to reclassification of LVEF compared with 10-beat analysis (online supplemental table 6).

\section{DISCUSSION}

This study has demonstrated that the index-beat method produces more reproducible quantification of systolic and diastolic left ventricular function in patients with AF than conventional averaging of consecutive beats, while saving time and without compromising validity. The within-beat coefficient of variability and intraobserver/interobserver reproducibility were all favourable using the index-beat approach to imaging in patients who have a variable stroke volume and filling time. Using the index-beat method routinely in clinical practice has the potential to improve workflow and productivity, enhance the reliability of echocardiography, and provide more confidence in the diagnosis and classification of heart failure in patients with AF.

Heart failure is common in patients with $\mathrm{AF}$, and accurate assessment of systolic and diastolic left ventricular function is essential for patient management. ${ }^{4}$ Although current guidelines recommend averaging 5-10 consecutive beats, ${ }^{6} 1415$ this is based on consensus opinion and lacks reliable evidence. ${ }^{16}$ Measuring consecutive beats is time-consuming and the overall measurement obtained will vary according to which beats are selected, making reliability in clinical practice uncertain. ${ }^{17}{ }^{18} \mathrm{AF}$ is characterised by a loss of atrial contraction and so ventricular filling relies heavily on the length of the R-R interval, with variation in intervals leading to considerable challenges to achieve reproducible measurements. ${ }^{19}$ In addition to cycle length, stroke volume is critically dependent on preload, and this is also variable in the setting of $\mathrm{AF}^{9}$

It is important to note the very high levels of variation we demonstrate using the guideline-recommended 10 consecutive-beat analysis; the CV of $41 \%$ for E/e' should be considered unacceptable, highlighting the limitations of current practice in patients with AF. The influence of the $\mathrm{R}-\mathrm{R}$ interval on contractility is believed to be caused by preload and uptake of calcium during the relaxation phase. The longer the R-R interval, the more time for calcium uptake and the greater the amount released in response to the sequential action potential, triggering a greater force of contraction. ${ }^{20}$ As ventricular filling and stroke volume for a particular beat are determined by the previous two R-R intervals, the index-beat method selects a cardiac cycle for analysis where the preceding and pre-preceding R-R interval are of similar duration. Hence, the real value of the indexbeat method may be to achieve a more physiologically appropriate measurement. In this context, the end diastolic volume should be similar and so the contractility will also be similar, producing less variability between index-beats when assessing systolic function. ${ }^{19} 2122$ The reliance on previous cycle lengths will also apply to GLS for detection of early myocardial dysfunction ${ }^{1723}$ and diastolic filling indices such as E/e'. 2425

Intraoperator reproducibility for both GLS and E/e' was shown to be highest when measuring on a single index-beat. Previous studies assessing strain rate have found a strong correlation between the index- beat and averages of 10 and 15 beats with high levels of agreement. ${ }^{1726}$ We also demonstrated that the index-beat method has a high level of reproducibility between different operators, an important contribution to value within the clinical setting, enhancing the practicality and usefulness of serial scans.

\section{Strengths and limitations}

A major concern with nearly all previous studies is the preselection of patients with 'good echocardiography windows' ${ }^{727}$ This is the first study to our knowledge in which the index-beat method has been interrogated in all patients with no pre-exclusions to image quality or heart rate, and all patients confirmed as being in AF at the time of echocardiography. The trial-based setting allowed us to 'double-blind' the imaging process; anonymised analyses were performed offline with a separate random code to the study identification number, and operators were blinded to patient details, clinical status and therapy. When compared with consecutive beats, our study showed no compromise in the correlation with NT-proBNP, a useful prognostic marker for both heart failure and coronary disease. ${ }^{28}$ However, this is clearly a limited analysis, and as the weak correlations between LVEF and NT-proBNP show, natriuretic peptide levels can be influenced by a variety of factors, including AF itself. ${ }^{29}$ When measured on an-index beat, LVEF was higher, on average, than using consecutive beats, and we also demonstrated that the index-beat led to reclassification of LVEF (online supplemental table 6). However, as we lack 'ground truth', further studies are warranted to compare the association of index-beat measurements with long-term clinical events in AF, and validation against invasive haemodynamic measurements. Our study was limited by nonsimultaneous acquisition of Doppler and chamber images, and although this is currently standard practice globally there are single-beat options available for $\mathrm{E} / \mathrm{e}$ ' that can also be valuable in $\mathrm{AF}^{30}$ With pressure on echocardiography services increasing due to growing patient populations and wider indications, the indexbeat method could increase the efficiency of echocardiography in AF; however, this was tested in a limited number of patients and only for E/e'. Qualitative assessment is also desirable to establish how to effectively introduce the index-beat approach in cardiology departments and the training required to aid clinical productivity. 


\section{CONCLUSION}

In a blinded analysis without preselection for image quality, assessing left ventricular function using the index-beat method provides a more reproducible and quicker method of assessing heart function in patients with AF. Pending independent validation, our results suggest that echocardiography departments should change to the index-beat method to diagnose and characterise heart failure in patients with AF.

\section{Key messages}

What is already known on this subject?

- Accurate assessment of ventricular function is essential to diagnose the type of heart failure in patients with atrial fibrillation (AF) and guide treatment strategies.

- However, measuring ventricular function in patients with AF is challenging due to the irregular cardiac cycle length.

\section{What might this study add?}

- The index-beat approach, whereby measurements of ventricular function are taken after two cycles with a similar $\mathrm{R}$-R interval, was compared with conventional averaging of consecutive beats.

- We demonstrated that the index-beat approach was more reproducible, more time-efficient and did not compromise clinical validity against natriuretic peptide levels.

\section{How might this impact on clinical practice?}

- Pending external validation, use of the index-beat in clinical practice could improve the assessment of ventricular function, leading to more precise treatment strategies for patients with $\mathrm{AF}$ and the potential for fewer hospital admissions and better patient quality of life.

\section{Author affiliations \\ ${ }^{1}$ Institute of Cardiovascular Sciences, University of Birmingham, Birmingham, UK ${ }^{2}$ Cardiology Department, University Hospitals Birmingham NHS Foundation Trust, Birmingham, UK \\ ${ }^{3}$ NIHR Birmingham Biomedical Research Centre, University Hospitals Birmingham NHS Foundation Trust and University of Birmingham, Birmingham, UK \\ ${ }^{4}$ Test Evaluation Research Group, Institute of Applied Health Research, University of Birmingham, Birmingham, UK \\ ${ }^{5}$ University of Birmingham Clinical Trials Unit, Birmingham, UK \\ ${ }^{6}$ Thrombosis Research Unit, Aalborg University, Aalborg, Denmark \\ ${ }^{7}$ Liverpool Centre for Cardiovascular Science, University of Liverpool and Liverpool Heart and Chest Hospital NHS Foundation Trust, Liverpool, UK \\ ${ }^{8}$ University Heart and Vascular Center, University Medical Center Hamburg- Eppendorf, Hamburg, Germany \\ ${ }^{9}$ Medical Sciences Division, University of Oxford, Oxford, UK \\ ${ }^{10}$ Deep Medicine, Nuffield Department of Women's and Reproductive Health, University of Oxford, Oxford, UK \\ ${ }^{11}$ NIHR Oxford Biomedical Research Centre, University of Oxford, Oxford, UK \\ ${ }^{12}$ Cardiology Clinical Academic Group - Molecular \& Clinical Sciences Institute, St George's University of London, London, UK \\ ${ }^{13}$ Patient and Public Involvement Team, Birmingham, UK \\ ${ }^{14}$ Institute of Cancer and Genomic Sciences, University of Birmingham, Birmingham, UK \\ ${ }^{15}$ Health Data Research (HDR)-UK Midlands, Birmingham, UK}

\section{Twitter Richard P Steeds @RichardSteeds}

Acknowledgements We are grateful to the patients who gave their time to take part in the RATE-AF trial. We would like to thank the trial team, particularly Patience Domingos (Research Nurse; Sandwell and West Birmingham Hospitals NHS Trust, Birmingham, UK), staff at the Wellcome/NIHR Clinical Research Facility (University Hospitals Birmingham NHS Foundation Trust, Birmingham, UK), the Birmingham Clinical Trials Unit (University of Birmingham, Birmingham, UK), and the Patient and Public Involvement Team (Mary Stanbury, Jacqueline Jones and Sandra Haynes). This paper presents independent research supported by the NIHR Birmingham Biomedical
Research Centre at the University Hospitals Birmingham NHS Foundation Trust and the University of Birmingham.

Contributors All authors are justifiably credited with authorship, according to the authorship criteria. In detail KVB, RPS and DK are responsible for overall content, conception and design; KVB, SG, KO, MG, JNT, RPS and PK for acquisition of data; KVB, DK, SM and AS for analysis and interpretation of data; KVB, DK, MS and RPS fo drafting the manuscript and final approval; and KVB, SG, AS, SM, KO, GL, PK, VYS, JC KR, MS, JNT, MG, AK, GVG, RPS and DK for revision of manuscript and final approval.

Funding KVB, DK and the RATE-AF trial were funded by a National Institute for Health Research (NIHR) Career Development Fellowship, awarded to DK (CDF-201508-074). The work is also supported by a British Heart Foundation (BHF) Accelerator Award to the University of Birmingham Institute of Cardiovascular Sciences (AA/18/2/34218). The opinions expressed are those of the authors and do not represent the NIHR, BHF or the UK Department of Health and Social Care.

Competing interests KVB reports she was the research fellow for the RATE-AF trial funded by a National Institute for Health Research (NIHR) Career Development Fellowship awarded to DK. SG: none directly relevant to this work; funding through the BigData@Heart Innovative Medicines Initiative (grant no 116074). GL: none directly relevant to this work; consultant for Bayer/Janssen, BMS/Pfizer, Medtronic, Boehringer Ingelheim, Novartis, Verseon and Daiichi Sankyo; and speaker for Bayer, BMS/Pfizer, Medtronic, Boehringer Ingelheim and Daiichi Sankyo; no fees are directly received personally. PK reports grants from NIHR, European Union and British Heart Foundation, during the conduct of the study; grants and non-financial support from the European Union, British Heart Foundation, Leducq Foundation, Medical Research Council (UK) and German Centre for Heart Research, from several drug and device companies active in atrial fibrillation, and has received honoraria from several such companies, outside the submitted work; in addition, PK is listed as inventor on two patents held by the University of Birmingham (Atrial Fibrillation Therapy WO 2015140571, Markers for Atrial Fibrillation WO 2016012783). JC: none directly relevant to this work; reports grants and personal fees from Bayer, Boehringer Ingelheim, Daiichi Sankyo and Pfizer/BMS; and personal fees from Medtronic, Boston Scientific and Abbott. KR reports grants from NIHR Oxford Biomedical Research Centre, University of Oxford; grants from UKRI's Global Challenge Research Fund (ESRC), PEAK Urban Programme Grant Ref: ES/P011055/1; grants from the British Heart Foundation (grant numbers: FS/19/36/34346 and PG/18/65/33872); grants from Oxford Martin School, University of Oxford, during the conduct of the study; and personal fees from PLOS Medicine and from BMJ Heart, outside the submitted work. DK reports grants from the National Institute for Health Research (NIHR CDF-201508-074 and NIHR HTA-130280), the British Heart Foundation (PG/17/55/33087 and $A A / 18 / 2 / 34218$ ), the European Society of Cardiology supported by educational grants from Boehringer Ingelheim/BMS-Pfizer Alliance/Bayer/Daiichi Sankyo/ Boston Scientific, the NIHR/University of Oxford Biomedical Research Centre and British Heart Foundation/University of Birmingham Accelerator Award (STEEER-AF NCT04396418), EU/EFPIA Innovative Medicines Initiative (BigData@Heart 116074) and IRCCS San Raffaele/Menarini (Beta-blockers in Heart Failure Collaborative Group NCT0083244), in addition to personal fees from Bayer (Advisory Board), AtriCure (Speaker fees), Amomed (Advisory Board), Protherics Medicines Development (Advisory Board) and Myokardia (Advisory Board), all outside the submitted work.

Patient consent for publication Patients have consented to publication of anonymised group results.

Ethics approval The trial received favourable ethical review from the UK Health Research Authority (East Midlands - Derby Research Ethics Committee; 16/EM/0178).

Provenance and peer review Not commissioned; externally peer reviewed.

Data availability statement Data sharing requests are required to meet the funder's policies; enquiries to be made to the corresponding author for Steering Committee review.

Supplemental material This content has been supplied by the author(s). It has not been vetted by BMJ Publishing Group Limited (BMJ) and may not have been peer-reviewed. Any opinions or recommendations discussed are solely those of the author(s) and are not endorsed by BMJ. BMJ disclaims all liability and responsibility arising from any reliance placed on the content. Where the content includes any translated material, BMJ does not warrant the accuracy and reliability of the translations (including but not limited to local regulations, clinical guidelines, terminology, drug names and drug dosages), and is not responsible for any error and/or omissions arising from translation and adaptation or otherwise.

Open access This is an open access article distributed in accordance with the Creative Commons Attribution 4.0 Unported (CC BY 4.0) license, which permits others to copy, redistribute, remix, transform and build upon this work for any purpose, provided the original work is properly cited, a link to the licence is given, and indication of whether changes were made. See: https://creativecommons.org/ licenses/by/4.0/

\section{ORCID iDs}

Karina V Bunting http://orcid.org/0000-0003-4602-4377 
Gregory YH Lip http://orcid.org/0000-0002-7566-1626

Paulus Kirchhof http://orcid.org/0000-0002-1881-0197

A John Camm http://orcid.org/0000-0002-2536-2871

Richard P Steeds http://orcid.org/0000-0001-5687-2535

Dipak Kotecha http://orcid.org/0000-0002-2570-9812

\section{REFERENCES}

1 Lane DA, Skjøth F, Lip GYH, et al. Temporal trends in incidence, prevalence, and mortality of atrial fibrillation in primary care. J Am Heart Assoc 2017;6:e05155.

2 Kotecha D, Lam CSP, Van Veldhuisen DJ, et al. Heart Failure With Preserved Ejection Fraction and Atrial Fibrillation: Vicious Twins. J Am Coll Cardiol 2016;68:2217-28.

3 Kotecha D, Piccini JP. Atrial fibrillation in heart failure: what should we do? Eur Heart J 2015;6:ehv513-3257.

4 Kirchhof P, Benussi S, Kotecha D, et al. 2016 ESC guidelines for the management of atrial fibrillation developed in collaboration with EACTS. Eur Heart J 2016;37:2893-962.

5 Cleland JGF, Bunting KV, Flather MD, et al. Beta-blockers for heart failure with reduced, mid-range, and preserved ejection fraction: an individual patient-level analysis of double-blind randomized trials. Eur Heart J 2018;39:26-35.

6 Lang RM, Badano LP, Mor-Avi V, et al. Recommendations for cardiac chamber quantification by echocardiography in adults: an update from the American Society of echocardiography and the European association of cardiovascular imaging. J Am Soc Echocardiogr 2015;28:1-39.

7 Kotecha D, Mohamed M, Shantsila E, et al. Is echocardiography valid and reproducible in patients with atrial fibrillation? A systematic review. Europace 2017;19:1427-38.

8 Bunting KV, Steeds RP, Slater LT, et al. A practical guide to assess the reproducibility of echocardiographic measurements. J Am Soc Echocardiogr 2019:32:1505-15.

9 Belenkie I. Beat-To-Beat variability of echocardiographic measurements of left ventricular end diastolic diameter and performance. J Clin Ultrasound 1979:7:263-8.

10 Kotecha D, Bunting KV, Gill SK, et al. Effect of digoxin vs bisoprolol for heart rate control in atrial fibrillation on patient-reported quality of life: the RATE-AF randomized clinical trial. JAMA 2020;324:2497-508.

11 Kotecha D, Calvert M, Deeks JJ, et al. A review of rate control in atrial fibrillation, and the rationale and protocol for the RATE-AF trial. BMJ Open 2017;7:e015099.

12 Zou GY. Sample size formulas for estimating intraclass correlation coefficients with precision and assurance. Stat Med 2012;31:3972-81.

13 Meng X-li, Rosenthal R, Rubin DB. Comparing correlated correlation coefficients. Psychol Bull 1992;111:172-5.

14 Mathew TSR, Jones R, Kanagala P. Wheeler R. a guideline protocol for the echocardiographic assessment of diastolic dysfunction. British Society of Echocardiography 2013 https://www.bsecho.org/media/112942/dd_protocol_final. pdf
15 Donal E, Lip GYH, Galderisi M, et al. EACVI/EHRA expert consensus document on the role of multi-modality imaging for the evaluation of patients with atrial fibrillation. Eur Heart J Cardiovasc Imaging 2016;17:355-83.

16 Wu VC-C, Otani K, Yang C-H, et al. Optimal number of Heartbeats required for representing left chamber volumes and function in patients with Rate-Controlled atrial fibrillation. J Am Soc Echocardiogr 2019;32:495-502.

17 Lee C-S, Lin T-H, Hsu P-C, et al. Measuring left ventricular peak longitudinal systolic strain from a single beat in atrial fibrillation: validation of the index beat method. J Am Soc Echocardiogr 2012;25:945-52.

18 Wang C-L, Ho W-J, Luqman N, et al. Biplane assessment of left ventricular function during atrial fibrillation at beats with equal subsequent cycles. Int I Cardiol 2006;113:54-60

19 Wang C-L, Lin K-H, Luqman N, et al. Simultaneous biplane single-beat assessment of left ventricular systolic function in patients with atrial fibrillation. Am J Cardiol 2004;94:942-4.

20 Eisner DA, Caldwell JL, Kistamás K, et al. Calcium and excitation-contraction coupling in the heart. Circ Res 2017:121:181-95.

21 Kerr AJ, Simmonds MB, Stewart RA. Influence of heart rate on stroke volume variability in atrial fibrillation in patients with normal and impaired left ventricular function. $A m$ J Cardiol 1998;82:1496-500.

22 Kong L-Y, Sun L-L, Chen L-L, et al. Value of index beat in evaluating left ventricular systolic and diastolic function in patients with atrial fibrillation: a dual Pulsed-Wave Doppler study. Ultrasound Med Biol 2020;46:255-62.

23 Nafati C, Gardette M, Leone M, et al. Use of speckle-tracking strain in preloaddependent patients, need for cautious interpretation! Ann Intensive Care 2018;8:29.

24 Kusunose K, Yamada H, Nishio S, et al. Clinical utility of single-beat E/e' obtained by simultaneous recording of flow and tissue Doppler velocities in atrial fibrillation with preserved systolic function. JACC Cardiovasc Imaging 2009:2:1147-56.

25 Tabata T, Grimm RA, Asada J, et al. Determinants of LV diastolic function during atrial fibrillation: beat-to-beat analysis in acute dog experiments. Am J Physiol Heart Circ Physiol 2004;286:H145-52.

26 Kusunose K, Yamada H, Nishio S, et al. Index-beat assessment of left ventricular systolic and diastolic function during atrial fibrillation using myocardial strain and strain rate. J Am Soc Echocardiogr 2012;25:953-9.

27 Bunting KV, O'Connor K, Steeds RP, et al. Cardiac imaging to assess left ventricular systolic function in atrial fibrillation. Am J Cardiol 2021;139:40-9.

28 Kotecha D, Flather MD, Atar D, et al. B-type natriuretic peptide trumps other prognostic markers in patients assessed for coronary disease. BMC Med 2019;17:72.

29 Chua W, Purmah Y, Cardoso VR, et al. Data-driven discovery and validation of circulating blood-based biomarkers associated with prevalent atrial fibrillation. Eur Heart J 2019:40:1268-76.

30 Li C, Zhang J, Zhou C, et al. Will simultaneous measurement of E/e' index facilitate the non-invasive assessment of left ventricular filling pressure in patients with nonvalvular atrial fibrillation? Eur J Echocardiogr 2010;11:296-301. 